\title{
Mathematical model for crane scheduling in mixed yard
}

\author{
Huihui Han ${ }^{1}$, Liying Mi², Tao Yang ${ }^{*}, 3$, and Xin Luo \\ ${ }^{1}$ School of Computer Science \& Technology, Donghua University, Shanghai, 201620, P.R. \\ China,+8618302166037, E-mail: 18790507445@163.com \\ ${ }^{2}$ Country School of Foreign Studies, University of Finance and Economics, Shanghai, 200433, \\ P.R.China. E-mail: mi.liying@mail.shufe.edu.cn \\ ${ }^{3}$ Donghua University Shanghai, 201620, P.R. China. E-mail: yangtao@dhu.edu.cn \\ ${ }^{4}$ School of Computer Science \& Technology, Donghua University, Shanghai, 201620, P.R. China. \\ Email:xluo@dhu.edu.cn
}

\begin{abstract}
In mixed yards, the stacking status of the container is not in the order of the shipment, causing the efficiency of the process of loading and unloading decreased. Therefore, it is very important to come up with a method using the existing facilities to improve the efficiency and reduce the cost. In this paper(Produces the permission block, and copyright information Permission to make digital or hard copies of part or all of this work for personal or classroom use is granted without fee provided that copies are not made or distributed for profit or commercial advantage and that copies bear this notice and the full citation on the first page. Copyrights for third-party components of this work must be honored. For all other uses, contact the owner/author(s).) We propose a method that sets a buffer in the yard to minimize the duration of the shifting process. To implement this method, the integer programming model is founded and the small scale accurate solution is used to implement the crane scheduling in the shifting process. The results showed that the model mentioned earlier can solve the problem of crane scheduling in the process of shifting with efficiency and provide strategic support for the management of yard.
\end{abstract}

\section{Introduction}

With the development of modern port logistics, the logistics activities continue to be dense, the container transport become continuous popularization, and the growth of the throughput of ports, the problem of insufficient resources has begun to surface in the space of yards has become increasingly prominent. To solve this very problem, the mixed model prevails, which means there are import and export containers in the same container terminal, at the same period of time. In mixed yards, management and scheduling of the cranes is of great importance. It is linked to the efficiency of container gate in and out, even the operation of the whole port.

\footnotetext{
* Tao Yang: yangtao@dhu.edu.cn
} 
Due to the inconsistency of the container storage status and shipping sequence, the efficiency of the loading process. This problem can be solved by taking the spare time before entering the yard to check the containers. This solution will not only improve the efficiency of stacking operations, but also reduce cost and improve satisfaction. In this paper, we are going to introduce the buffer area of the yards, take the minimum time to finish all requests as the goal, then discuss and optimize the yard crane scheduling problem in mixed yard.

\section{Literature review}

Many researchers have studied the stacking operations and crane scheduling in mixed yard. Liang et al. [1] studied multiple cranes working on containers loading and discharging operations in mixed yards. Taking the safe distance among yard cranes, equipment interference and the continuity into consideration, and then established a mixed integer mathematical model, to minimize the job completion time and reduce delay. Shao et al. [2] divided the yard crane scheduling problem into moving path and relocation scheduling. They developed a polynomial algorithm to get an optimal solution of the yard crane moving path model, and a bi-level heuristic algorithm based on memory simulated annealing to obtain satisfying solutions of the relocation optimization model. Zheng et al. [3] studied the crane scheduling in mixed yards, aiming at minimizing the cost of all tasks and the nonload and non-unload cost of all operating yard cranes during the fixed span, through nonlinear mathematical planning model, and solve it with mixed genetic algorithm and tabu searching algorithm. Zheng et al. [4] studied the optimization of crane scheduling in mixed yard, taking the priorities and deadlines of both external and internal trucks into account, aiming at minimizing the operational cost and relocation amount. A genetic algorithm integrated the solution space cutting function and constructed tactics are proposed to solve the problem. Amir et al. [5] studied the effect of a handshake area on the performance of twin automated stacking cranes. They set up a model to minimize the completing time and blocking time, and then set 4 decision variables for the heuristic scheduling algorithm, despite the final result not being so idea.

Many scholars did some deep research on the shifting operations in mixed yards. To solve the problem of relocation, Zhu et al. [6] constructed a searching strategy with minimum number of basic relocation, yard crane cost and number of relocation times, then the beam search algorithm was used as well. Dong et al. [7] studied the optimum scheduling problem of container yard relocation among multiple bays. They established a mathematical model based on the balance between utilization rate and the relocating rate, and then solved the problem with mixed heuristic algorithm. Bian et al. [8] studied the problem of container preloading in the exporting area. A two-staged algorithm consists of a neighborhood searching algorithm and an integer programming model is proposed to develop a pre-marshalling plan to improve the layout of containers in a bay. Zhou et al [9] established a simulation model of the reshuffling process based on analyzing the actual reshuffles of import containers in the container yard. The heuristic algorithm is designed basing on the total least-reshuffles-estimate rule.

\section{Yard crane scheduling model}

\subsection{Description}

In mixed yards, to improve the efficiency of the containers' loading and unloading process, usually the containers are shifted before the arrival of a ship. Different from the traditional 
container yards, there are import and export containers in the yard at the same time, which means crane scheduling process will get harder. The problem will be described as follows. Two cranes in the same container terminal with a buffer in the middle of it. Shifting requests are completed by two yard cranes. The cranes do not go through the buffer zone. When a shifting request spans the buffer, the container is transported from the starting position to the buffer by a crane, and the other crane moves it from the buffer to the targeting position.

The optimization goal is to minimize the completing time of all requests, which assumes that: (1) the shifting is carried out only in the container sector, the storage status and the shifting demand are already known; (2) the container properties are all the same, special containers are not taken into consideration; (3) no new container entering the yard during the shifting process; (4) assuming the buffer is in the middle of the container terminal, which occupies one bay.

\subsection{Model construction}

\subsubsection{Parameters and variables.}

(1) indicator set

$\mathrm{i}, \mathrm{j}$ : request indicators;

$\mathrm{k}$ : yard crane indicators;

m: number of yard crane;

(2) parameters

M: number of yard cranes;

$\mathrm{N}$ : number of requested containers;

$\mathrm{n}$ : number of requested containers which need to be split;

nl: number of request which completed by the land side yard crane;

ns: number of request which completed by the sea side yard crane;

$\Omega$ : number of requested containers, $\mathrm{N}$ task containers matching the natural number of $(1-\mathrm{N})$;

$\mathrm{H}$ : maximum number of secure stacks;

$\rho$ : spreader landing and grab (or put down and lift) a container's time consuming;

$\mathrm{D}$ : minimum safe distances between yard cranes;

$K_{m}$ : number of shifting request which completed by yard crane m;

1: the length of a single bay;

$\mathrm{v}$ : the processing speed of the yard crane;

(3) decision variables

$X_{i m}$ : number of the containers which were handled by the bridge $\mathrm{m} \mathrm{Nth}$;

$a\left(X_{i m}\right)$ : moving path of the extraction of container $X_{i m}$;

$H\left[a\left(X_{i m}\right)\right]$ : maximum stacking height that occurs during the execution of $a\left(X_{i m}\right)$;

$P_{k t}$ : yard crane's position $\mathrm{k}$ at time $\mathrm{t}$;

$T_{l}$ : completing time of land side yard crane;

$T_{S}$ : completing time of sea side yard crane.

\subsubsection{Model building.}

Mark the coordinates of the container respectively $\left(b_{i}, s_{i}, t_{i}\right)$ and $\left(b^{\prime}{ }_{i}, s^{\prime}{ }_{i}, t^{\prime}{ }_{i}\right)$ before and after shifting. According to the analysis above, the mathematical model of shifting crane scheduling problem is constructed as follows:

Objective function: 


$$
\mathrm{T}=\{\mathrm{TL}, \mathrm{TS}\}
$$

Constraint condition:

$$
\begin{gathered}
B_{i} \geq\left(b^{\prime}{ }_{i}-b_{i}\right)+\left(b_{i+1}-b^{\prime}{ }_{i}\right), 1 \leq \mathrm{i} \leq \mathrm{N}(2) \\
B_{i} \geq\left(b_{i}-b^{\prime}\right)+\left(b^{\prime}{ }_{i}-b_{i+1}\right), 1 \leq \mathrm{i} \leq \mathrm{N}(3) \\
B_{i} \geq\left(b^{\prime}{ }_{i}-b_{i}\right)+\left(b^{\prime}{ }_{i}-b_{i+1}\right), 1 \leq \mathrm{i} \leq \mathrm{N}(4) \\
B_{i} \geq\left(b_{i}-b^{\prime}{ }_{i}\right)+\left(b_{i+1}-b^{\prime}{ }_{i}\right), 1 \leq \mathrm{i} \leq \mathrm{N}(5) \\
T_{l} \geq \sum_{i=1}^{n l+n} B_{i} * l \div v+4 \rho, 1 \leq \mathrm{i} \leq \mathrm{N}, 1 \leq \mathrm{nl}, \mathrm{n} \leq \mathrm{N}(6) \\
T_{s} \geq \sum_{i=1}^{n s+n} B_{i} * l \div v+4 \rho, 1 \leq \mathrm{j} \leq \mathrm{N}, 1 \leq \mathrm{ns}, \mathrm{n} \leq \mathrm{N}(7) \\
\mathrm{N}=\mathrm{nl}+\mathrm{ns}+\mathrm{n}, 1 \leq \mathrm{nl}, \mathrm{ns}, \mathrm{n} \leq \mathrm{N} \\
P_{k t} \leq P_{(k+1) t}-D, 1 \leq \mathrm{k} \leq \mathrm{M}(9) \\
\mathrm{h}\left[\mathrm{a}\left(X_{i m}\right)\right] \leq \mathrm{H}, 1 \leq \mathrm{i} \leq \mathrm{N}, 1 \leq \mathrm{k} \leq \mathrm{M}(10) \\
\sum_{m=1}^{M} K_{m}=N, 1 \leq \mathrm{k} \leq \mathrm{M}(11) \\
X_{i m} \in \Omega, 1 \leq \mathrm{i} \leq \mathrm{N}, 1 \leq \mathrm{m} \leq \mathrm{M}(12) \\
\mathrm{a}\left(X_{i m}\right) \in \mathrm{A}\left(X_{i m}\right), 1 \leq \mathrm{i} \leq \mathrm{N}, 1 \leq \mathrm{m} \leq \mathrm{M}(13) \\
K_{m} \leq N-1,1 \leq \mathrm{m} \leq \mathrm{M}(14)
\end{gathered}
$$

The objective function (1) indicates that when two cranes complete the same one request together, the completing time of the request is set by the larger one; Constraints (2) - (5) indicate the bays that crane completing one single shifting request of a container and then moves to the next position; Constraints (6) and (7) represent the time that cranes on land and at sea need to complete all the requests; Constraints (8) represent the relationship between the number of requests completed by the sea side crane alone, the number of requests completed by the land side crane alone and the number of requests which need to be split and completed by two cranes; Constraints (9) represent the safe distance between two cranes and ensure the safety of operations; Constraints (10) makes sure that the stack height during each operation is less than the maximum stack height; Constraint (11) to ensure that all tasks completed; Constraints (12) - (14) specify the range of values for each variable.

\subsection{Case study}

To verify the feasibility of the model, we will solve an example as the following one. In the given example, there are six requests, which are completed by two cranes, as shown in Table 1. For the sake of simplicity, assuming the travel duration in bay (Y-direction) is longer than the ones in stack (X-direction), so only the movement time in bay is taken into consideration.

We simplified the shifting movement into four steps:

(1) The crane moves to the starting position of a request;

(2) The spreader goes down and grabs the container, then raise to the safe height;

(3) The crane moves to the target position requested;

(4) The spreader puts the container to destination and rises.

Repeat steps (1) - (4) until all shifting requests are completed. According to the actual cranes' working data, 10 seconds will be consumed when a crane goes through a single bay. One single going down and grabbing (or releasing) the container would consume 30 seconds. Figure 1 shows the moving path of the crane both with and without buffer zone. 
Table 1. Shifting requests' sequence

\begin{tabular}{ccc|ccc}
\hline \multicolumn{3}{c|}{ without buffer zone } & \multicolumn{3}{c}{ with buffer zone } \\
\hline start position & $\begin{array}{c}\text { target } \\
\text { position }\end{array}$ & $\begin{array}{c}\text { number of } \\
\text { crane }\end{array}$ & start position & $\begin{array}{c}\text { target } \\
\text { position }\end{array}$ & $\begin{array}{c}\text { number of } \\
\text { crane }\end{array}$ \\
\hline 32 & 10 & 2 & 32 & 16 & 2 \\
1 & 5 & 1 & 16 & 10 & 1 \\
28 & 32 & 2 & 28 & 5 & 1 \\
25 & 32 & 2 & 25 & 32 & 2 \\
6 & 1 & 1 & 6 & 1 & 2 \\
1 & 20 & 1 & 1 & 16 & 1 \\
& & & 16 & 20 & 2 \\
\hline
\end{tabular}

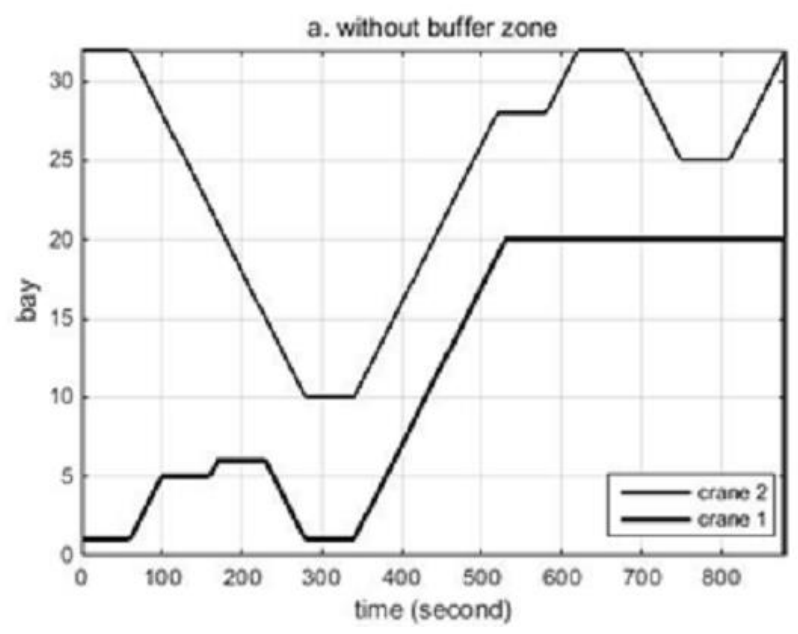

Fig.1 (a): Move path of cranes without buffer zone

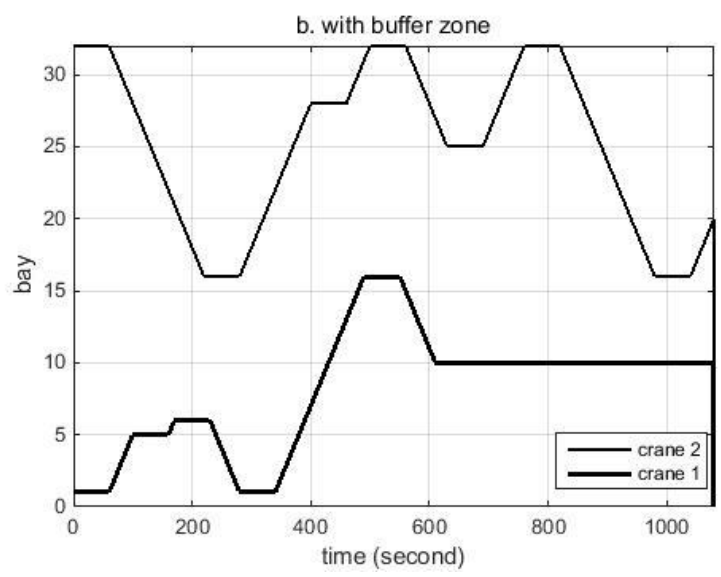

Fig.1 (b): Move path of cranes with buffer zone 
The moving path of the cranes without buffer zone is shown in Fig. 1 (a). Both land side crane and sea side crane are moving in the entire yard space, and crane 1 would have to wait for a long time after completing its requests, requests of the cranes are imbalance, this is wasting the resources of crane yards. The moving path of cranes with buffer zones is shown in Fig. 1 (b). The cranes are only working on one side of the yard or buffer zone. The probability of generating interferences is relatively small. Although there is also waiting time, the probability of waiting time is reduced than the former one.

\section{Conclusion}

In this paper, we would be focused on a crane yard with buffer. Through the analysis of the paper, we get the shifting request sequence through the nearest neighbor equation, and the cranes' priority is determined by the longest remaining time. However, this article mentioned that the yard working condition is static and there is no new container entering the yard in the case of any shifting operation, so the next step is to solve the shifting problem with a dynamic situation.

Acknowledgments: The authors would like to thank Guangdong Province collaborative innovation and platform Environmental Science build of special funds (2014B090908004); Dongguan City professional town innovation service platform construction project" Dongguan City Humen garment Collaborative Innovation Center", whose constructive comments and suggestions helps us to improve the quality of this paper.

\section{References}

[1] Liang Chengji, Zhai diandian, and Wang dandan. 2016. Dynamic Scheduling Model Research for Multiple Yard Crane in Container Terminal. J. Computer Simulation. 33, 3 (Mar. 2016), 363366.(in Chinese)

[2] Shao Qianqian, Xu Tingting, Yang Huiyun, and Jin Zhihong. 2016. Scheduling Optimization of Yard Crane for Import Conrainers Based on Truck Batch Arrival Pattern. J. Control and Decision. 31, 9 (Sep. 2016), 1654-1662. (in Chinese)

[3] Zheng Hongxing, and Yu Kai. 2013. Yard Crane Scheduling in the Mixture Storage Block Based on Hybrid Genetic Algorithm. J. Journal of Transportation Systems Engineering and Information Technology. 13, 5 (Oct. 2013), 150-158. (in Chinese)

[4] Zheng Hongxing, Dong Yiwen, Kuang Haibo, and Yu Kai. 2016. Yard Cranes Scheduling with Relocation at a Mixed Storage Block for Ship Loading. J. Systems Engineering - Theory \& Practice. 36, 9 (Sep. 2016), 2362-2373. (in Chinese)

[5] Amir Hossein Gharehgozli, Floris Gerardus Vernooij, and Nima Zaerpour. 2017. A simulation study of the performance of twin automated stacking cranes at a seaport container terminal. J. European Journal of Operational Research. (Feb. 2017), 108-128.

[6] Zhu Minghua, Cheng Huanchong, and Fan Xiumin. 2012. Blocks Relocation problem with filter based beam search algotithm. J. Computer Integrated Manufacturing Systems. 18, 3 (Mar. 2012), 639-644. (in Chinese)

[7] Dong Xiaomei, Pan Yu, Xu Xiaoyong, and Ding Yanyan. 2012. Mathematical Model and Hybrid Heuristic Optimization Algorithm of Container Rehandling Among Different Bay Locations. J. Operation Research and Management Science. 21, 6 (Dec. 2012), 112-118. (in Chinese)

[8] Bian Zhan, Li Na, Li Xiangjun, and Jin Zhihong. 2014. Hybrid Optimization Algorithm for Pre-marshalling Export Containers. J. Control and Decision. 29, 2 (Feb. 2014), 373-378. (in Chinese) 
[9] Zhou Pengfei, and Li Pian. 2013. Simulation Analysis and Heuristic Algorithm for Reshuffle Optimazation of Import Containers in Container Terminal. J. Systems Engineering - Theory \& Practice. 33, 12 (Dec.2013), 3145-3155. (in Chinese) 